\title{
Genetic Mapping of Variation in Spatial Learning in the Mouse
}

\author{
Daniela Steinberger, ${ }^{1,2 \star}$ David S. Reynolds, ${ }^{3 \star}$ Pushpindar Ferris, ${ }^{3}$ Rachael Lincoln,,${ }^{3}$ Susmita Datta, ${ }^{1}$ Joanna Stanley, ${ }^{3}$ \\ Andrea Paterson, ${ }^{3}$ Gerard R. Dawson, ${ }^{3}$ and Jonathan Flint ${ }^{1}$ \\ ${ }^{1}$ Wellcome Trust Centre for Human Genetics, Oxford, OX3 7BN, United Kingdom, ${ }^{2}$ Bioscientia, Center for Human Genetics, 55218 Ingelheim, Germany, \\ and ${ }^{3}$ Merck Sharp and Dohme Research Laboratories, The Neuroscience Research Centre, Terlings Park, Essex, CM20 2QR United Kingdom
}

\begin{abstract}
Inbred strains of mice are known to differ in their performance in the Morris water maze task, a test of spatial discrimination and place navigation in rodents, but the genetic basis of individual variation in spatial learning is unknown. We have mapped genetic effects that contribute to the difference between two strains, DBA/2 and C57BL6/J, using an F2 intercross and methods to detect quantitative trait loci (QTL). We found two QTL, one on chromosome 4 and one on chromosome 12, that influence behavior in the probe trial of the water maze (genome-wide significance $p=0.017$ and 0.015 , respectively). By including tests of avoidance conditioning and behavior in a novel environment, we show that the QTL on chromosomes 4 and 12 specifically influence variation in spatial learning. QTL that influence differences in fearful behavior (on chromosomes 1,3,7,15, and 19) operate while mice are trained in the water maze apparatus.
\end{abstract}

Key words: quantitative trait locus; spatial learning; mouse genetics; water maze; genetic mapping; conditioned fear

\section{Introduction}

The effect of genetic mutations on spatial learning has been studied extensively using transgenic techniques (Chen and Tonegawa, 1997), but there have been fewer attempts to identify the genetic variants responsible for differences in spatial learning between inbred strains of mice (Crawley et al., 1997), variants that may well occur in a different set of genes from those so far subjected to transgenic analysis. For instance, genetic mapping performed in an inbred strain cross detected almost no overlap in the chromosomal location of known mutants and genes affecting variation in circadian rhythm (Shimomura et al., 2001). A similar result for the analysis of spatial learning would open a pathway for the eventual identification of novel genes involved in learning and memory processes. Although the molecular characterization of loci influencing phenotypic variation in inbred strain crosses has proved to be more difficult than initially anticipated (Flint and Mott, 2001), progress in genome sequence projects and the availability of complete gene catalogs, together with novel genetic mapping strategies, suggest that gene identification will soon become feasible.

The Morris water maze task has been widely used as a test of spatial discrimination and place navigation in rodents (D'Hooge and De Deyn, 2001). The task is sensitive to alterations in the function of the hippocampal formation, a region of the brain the pathology of which is implicated in a number of human brain disorders including Alzheimer's disease and age-dependent memory loss. However, performance in the water maze requires relatively good sensory and motor facility to acquire spatial learn-

\footnotetext{
Received Sept. 20, 2002; revised Nov. 14, 2002; accepted Dec. 11, 2002

This work was funded by a Medical Research Council LINK award in collaboration with Merck Sharp and Dohme and by the Wellcome Trust (J.F., A.B., A.N., R.M.).

${ }^{*} D$.S. and D.S.R. contributed equally to this work.

Correspondence should be addressed to Dr. Jonathan Flint, Wellcome Trust Centre for Human Genetics, Roosevelt Drive, 0xford, 0X3 7BN, UK. E-mail: jf@molbiol.ox.ac.uk.

Copyright $\odot 2003$ Society for Neuroscience $\quad 0270-6474 / 03 / 232426-08 \$ 15.00 / 0$
}

ing, and performance is affected by motivational and emotional variation. In a strain comparison between 129S2/Sv and C57BL/ $6 \mathrm{~J}$, Contet and colleagues (2001) showed that differences in latencies to reach the hidden platform during the training period reflected differences in swim speed. Differences in water maze performance between inbred strains of mice, or between mice of different genotypes, are likely to arise from a combination of different psychological processes, rather than representing purely variation in spatial learning. Consequently, without including adequate controls for confounds, genetic mapping of behavioral variation in the water maze may identify loci that have no relevance to spatial learning.

Here we report the results of a genetic mapping study of an F2 intercross between DBA/2 (poor performer) and C57BL/6J (good performer). Our experiment included tests for other factors likely to influence variation in water maze performance. In particular we were concerned that response to stress, imposed by the novelty of the apparatus and the need to swim, would differentially affect spatial learning ability. Water maze learning is aversively motivated behavior, and several studies have demonstrated that performance is affected in stressed animals (Holscher, 1999). Because genetic effects also determine variation in fearfulness in inbred strain crosses (Caldarone et al., 1997; Wehner et al., 1997; Turri et al., 2001a), it was essential to control for this confound in our mapping study. Therefore we included a test that measures an animal's ability to learn to avoid a stressful stimulus (mild foot-shock) and a test of an animal's response to a novel environment (behavior in an open-field apparatus). We used the conditioned avoidance test because it is a nonhippocampal-dependent test of learning that uses stress as a motivator for learning (Gray and McNaughton, 1983). We hypothesized that QTL influencing emotional behavior would be common to both water maze and avoidance tasks, but that separate loci would influence performance in hippocampal and nonhippocampal-dependent tasks. 
a

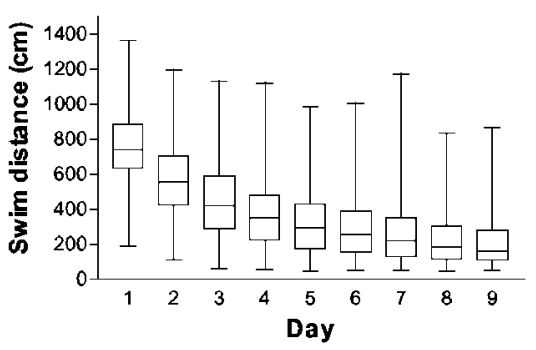

b

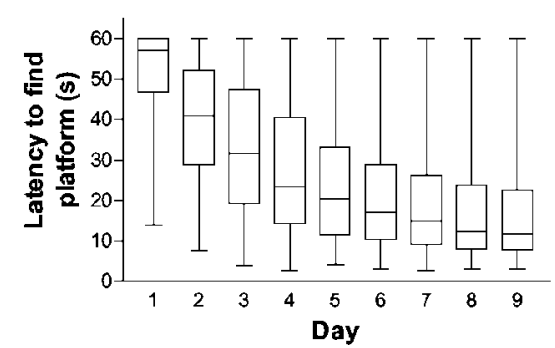

C

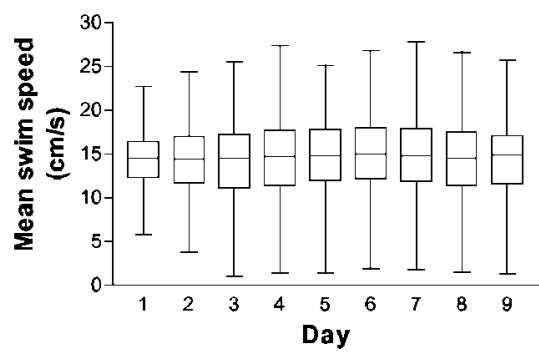

Figure 1. Box and whisker plots of daily performance of mice in the hidden platform water maze task. Over the $9 \mathrm{~d}$ of training mice showed a decrease in swim distance ( $a$ ) and latency to reach the hidden platform $(b)$, suggesting that they were learning the platform location. The mean swim speed $(c)$ did not change throughout the training period.

\section{Materials and Methods}

F2 intercross. An F2 generation of 396 male mice was used, derived from a cross between C57BL6/J and DBA2/J. Mice were maintained under controlled conditions of humidity $(55 \pm 10 \%)$ and temperature $(21 \pm$ $1^{\circ} \mathrm{C}$ ), a $12 \mathrm{hr}$ cycle (lights on at 7:00 A.M. and off at 7:00 P.M.), with ad libitum access to food and water. All mice were singly housed and weighed 20-25 gm at the start of experiments. The experimental sequence was as follows: open field ( $1 \mathrm{~d}$ in week 1 ), conditioned avoidance (Monday-Friday in week 2), and finally water maze (Monday-Friday in weeks 3 and 4). There were at least $2 \mathrm{~d}$ between each test.

Open field. Each mouse was placed in a square white Perspex open field arena $(50 \times 50 \mathrm{~cm})$ that was divided into a $5 \times 5$ grid by black lines. The arena was evenly illuminated by fluorescent lighting surrounding the four sides of the arena. The mouse was allowed to explore for $10 \mathrm{~min}$, and a video tracking system (HVS Image, Hampton, UK) was used to record the animal's movement. The arena was wiped clean between each mouse. The total distance covered in the 10 min test, time spent in the center, and number of entries into the center of the open field were recorded.

Conditioned avoidance. Mice were trained in two-compartment shuttle boxes controlled by a computer described in Dawson et al. (1999). Briefly, there was a $5 \mathrm{~min}$ habituation time at the beginning of each training session in which both house lights were switched off. There then followed 15 avoidance trials. Each trial began by detecting which side of the box the mouse was in and then illuminating that house light $(3 \mathrm{~W}, 24$ $\mathrm{V})$ for up to $10 \mathrm{sec}$. After that time, a $0.4 \mathrm{~mA}$ foot-shock was delivered through the grid floor for up to $10 \mathrm{sec}$. If the mouse moved to the other side of the box during the first $10 \mathrm{sec}$ of light presentation, an avoidance response was recorded, and the light was switched off. If the mouse moved during the shock period, an escape response was recorded, and both the shock and light were switched off. If the mouse did not move to the other side at all during the whole trial, an unmoved response was recorded, and the light and shock were terminated. There then followed a random intertrial interval $(26-40 \mathrm{sec})$ before the next trial began. Mice were trained for 5 consecutive days with one session per day.

Water maze. Mice were tested in a $1.0 \mathrm{~m}$ diameter circular pool [described in detail in Dawson et al. (1999)]. Mice were trained for $9 \mathrm{~d}$ (four trials per day) on a reference-memory hidden-platform acquisition task. Each trial consisted of placing the mouse in the pool at one of four start positions $90^{\circ}$ apart around the edge of the pool and allowing the mouse to swim to the hidden platform. If the mouse had not found the platform after $60 \mathrm{sec}$, it was placed on the platform by the experimenter. The mouse was allowed to remain on the platform for $30 \mathrm{sec}$ before being removed to an opaque high-sided plastic chamber for $30 \mathrm{sec}$. The next trial was then performed. For each trial, the latency to reach the platform, distance covered, and mean swim speed were recorded via video capture and image analysis using HVS Image water maze software. The data for each day were averaged over the four trials before being used for statistical analysis. On the 10th day a single 60 sec probe trial was run in which the platform was removed from the pool. The amount of time spent in each of the four quadrants of the pool and the number of times the mouse crossed the platform location were recorded. After the probe trial, four visible platform trials were performed (with the platform in the side of the pool opposite its location during hidden platform training) to check the vision of all mice.

Genotyping. On completion of the phenotyping, 391 of the original cohort of 396 mice were available for genotyping. DNA was extracted from tails and genotyped using standard techniques [as described in Fernandez-Teruel et al. (2002)]. Because all mice were male, we have not mapped the $\mathrm{X}$ chromosome. We chose markers from the radiation hybrid (RH) map to provide intermarker intervals of between 20 and 30 centimorgan (cM) intervals. The order of all markers was determined using the MAPMAKER software package (Lincoln et al., 1992), and results were compared with radiation hybrid maps.

Statistics. Multivariate analyses were performed using Multi-QTL (Korol et al., 2001) and QTL-CARTOGRAPHER (JZmapqtl) (Basten et al., 1994). Significance levels were evaluated by permutation performed in the MultiQTL package (Korol et al., 2001). Contributions of a trait to a logarithm of the ratio of the odds in favor of linkage (LOD) score were estimated in the Multi-QTL package, as described previously (Fernandez-Teruel et al., 2002). Composite interval mapping (Zeng 1994) was performed using QTL-CARTOGRAPHER (Basten et al., 1994).

\section{Results}

\section{Water maze task}

Over the $9 \mathrm{~d}$ of water maze trials there was a reduction in the mean distance traveled and the latency to reach the hidden platform, indicating a mean improvement in the animals' performance (Fig. 1a,b). As expected, speed remained constant over the training period (Fig. 1c). In the probe trial, animals spent more time in the quadrant that contained the hidden platform (quadrant one) and passed more often through the location of the platform (Fig. 2). All mice learned to swim to the visible platform during the four visible trials, indicating adequate visual acuity to perform this task using the spatial cues.

To provide an individual measure of an animal's ability to learn, we calculated a $t$ statistic for each animal by regression. We took a logarithmic transformation of time (the day on which the animals were tested) and fitted a linear model in which each phenotype collected during the acquisition period of the water maze task (latency, distance, and speed) was regressed onto the time at which the measure was taken. Mean values of the $t$ statistics were -3.98 (SD 2.01) for distance, -4.45 (SD 2.56) for latency, and -0.03 (SD 2.40) for speed. Individual latency and distance $t$ statistics were used in subsequent genetic mapping experiments.

\section{Conditioned avoidance and open field}

Conditioned avoidance was assessed over $5 \mathrm{~d}$ for all animals. At the end of this time there was an increase in the mean number of avoidances and a corresponding decrease in escape and freezing responses (Fig. 3). These data demonstrate acquisition of a conditioned avoidance response. 
a

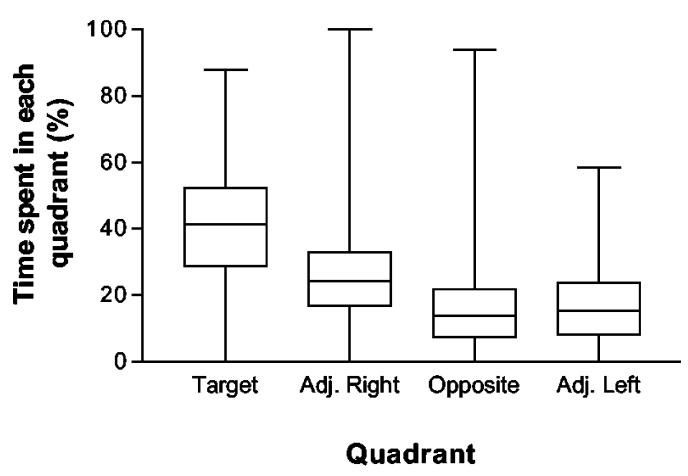

b

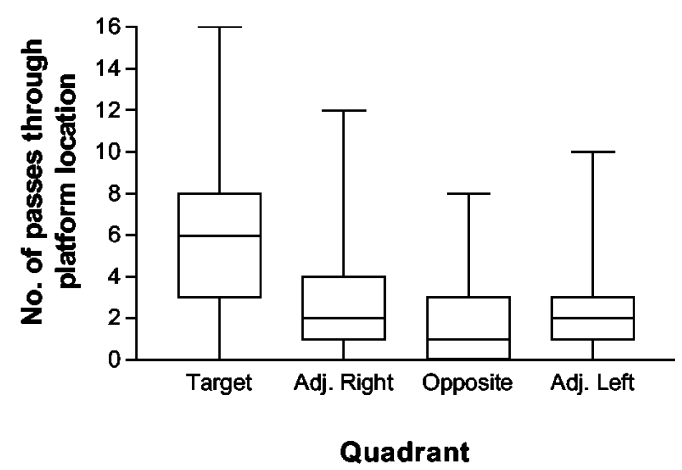

Figure 2. Box and whisker plot of probe trial performance in the water maze. Mice spent significantly more time (Student-Newman-Keuls post hoc test; $p<0.05$ ) in the quadrant where the platform had been (target quadrant) than in the other three quadrants ( $a$ ). Mice also made significantly more (Student-Newman-Keuls post hoc test; $p<0.05$ ) passes through the platform location in the target quadrant than through the equivalent position in the other three quadrants (b).

Analysis of the open field data showed that on average the animals spent $\sim 80 \%$ of their time in the edge squares of the arena and ventured out into the center for only the other $20 \%$ of the time. Furthermore, they spent $\sim 40 \%$ of their time in the corner squares of the arena, which are the least exposed areas of the open field arena. The time spent in these areas is typical of rodents displaying anxious behaviors in an unfamiliar exposed environment.

\section{Genetic mapping}

We used multitrait analysis to detect genetic effects on measures of spatial learning and on behavioral variation in the open field and conditioned avoidance tasks. In most circumstances, multivariate analysis is expected to increase the power to detect genetic effects as well as provide improved mapping resolution (Korol et al., 2001). We were able to detect QTL at genome-wide levels of significance, which influenced variation in joint measures, but none of these loci attained comparable significance in univariate analyses (Table 1).

We mapped jointly two measures taken on the probe trial of the water maze: the time spent in the quadrant that contained the hidden platform and the number of passes through the platform's location. The joint analysis revealed two loci on chromosomes 4 and 12 that exceeded the 5\% threshold. We then mapped measures of the rate of learning, using the $t$ statistics for distance traveled and latency to reach the hidden platform. Again we identified loci on chromosomes 4 and 12 and one additional locus on chromosome 8 . Finally, we performed a joint analysis of measures taken during the probe trial, adding the $t$ statistics for latency and distance traveled to those phenotypes already mapped. Inclusion of the additional phenotypes increased the evidence in favor of QTL on chromosomes 4 and 12. Figure 4 shows LOD plots for the measures taken in the water maze, and Table 2 gives the maximum LOD scores obtained and their significance, as derived by permutation.

In the open field, we jointly mapped activity, number of entries to the center of the apparatus, and time spent in the periphery of the arena. QTL on chromosomes 3, 7, 15, and 19 exceeded a genome-wide significance threshold of 5\% (Table 2). For conditioned avoidance we mapped variation in avoidance response, transitions, and escapes after $5 \mathrm{~d}$ of training; only one QTL, at the telomeric end of chromosome 1, exceeded the 5\% threshold. Figures 5 and 6 show LOD plots across the genome for the multivariate analyses, and Table 2 gives the significance of the LOD scores.

Although these results demonstrate a genetic separation of spatial learning from the acquisition of an avoidance response and behavior in a novel environment, they do not rule out a pleiotropic contribution from QTL on chromosomes 4 and 12 to other traits. We attempted to detect such effects by analyzing all phenotypes simultaneously and then assessing the significance of the contribution of a locus for the detection of QTL (Korol et al., 2001). Korol's method (Korol et al., 2001) reshuffles the individual values of each phenotype relative to the other traits and genotypes, and the resulting data set is reanalyzed. Then, over a large number of permuted data sets for each phenotype, the proportion of analyses is calculated in which the estimated QTL effect is greater than or equal to the QTL effect obtained with unpermutated data. The procedure is applied in a stepwise manner, excluding the insignificant traits by creating a new data set without them and repeating the permutation. It should be noted that the statistic only gives a measure of the contribution of the individual phenotype to the LOD score at a QTL detected using multivariate analysis; the results cannot be used to infer that a genetic effect influencing a single measure would be detectable with genomewide significance.

The new joint analysis did not detect any additional QTL. We then asked whether any of the QTL that influenced conditioned avoidance and open-field measures were making a contribution to the LOD score of water maze tasks. Using Korol's method to assess the contribution of each phenotype to the effect on chromosomes 4 and 12 (Korol et al., 2001), we found no evidence against specificity of action. On chromosome 4, the only significant contribution to the LOD score came from the number of passes through the position of the hidden platform, whereas on chromosome 12 this phenotype and the proportion of time spent in the quadrant with the platform were both significant.

Further evidence for the action of QTL on chromosomes 4 and 12 on spatial learning comes from the univariate analyses. We expect that the genetic effect from these loci would become apparent once the animals were trained, so we compared the LOD scores over the $9 \mathrm{~d}$ of the water maze experiment. Tables 3 and 4 show the results. The highest LOD score shown is 4.2 on chromosome 5, which was on day 1 for distance traveled. Once corrections are made for the number of phenotypes and testing on multiple occasions, this score fails to reach statistical significance. However, we are concerned here not with the significance of any single LOD score but whether there is a trend in the change of LOD scores over time.

We found that LOD scores on chromosomes 4 and 12 in- 
a

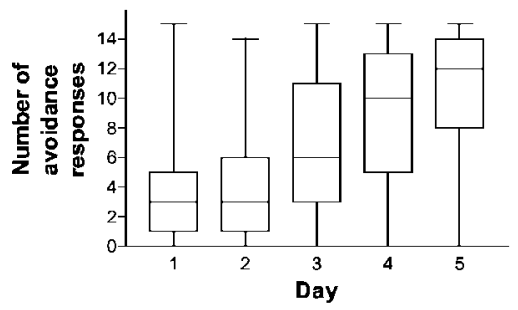

b

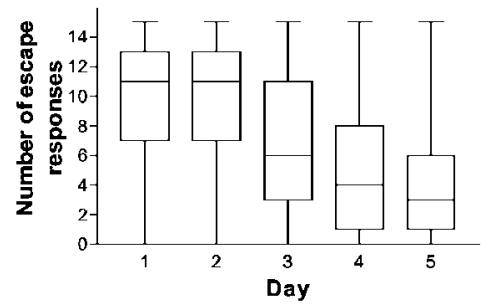

C

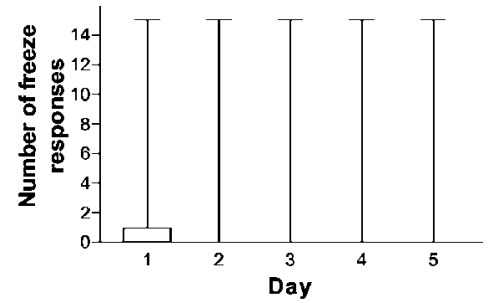

Figure 3. Box and whisker plots of daily performance in the conditioned avoidance test. Over the $5 \mathrm{~d}$ of training most mice learned to avoid the electric shock by moving to the other side of the chamber, as shown by the increase in avoidance responses ( $a$ ). Correspondingly, the number of responses in which the animal escaped to the other side after the shock had begun decreased with training $(b)$. Very few mice did not learn to either avoid or escape the shock, as indicated by the low number of freeze responses $(c)$.

\section{Table 1. LOD scores for univariate analyses}

\begin{tabular}{|c|c|c|c|c|c|c|}
\hline Phenotype & $\mathrm{Chr}$ & Lod & Pos. & Additive Effect & Dominance Effect & \% Variance \\
\hline \multicolumn{7}{|l|}{ Watermaze acquisition } \\
\hline Distance & 4 & 2.0 & 30 & 1.4 & 1.5 & 2.4 \\
\hline Latency & 4 & 2.2 & 30 & 1.2 & 1.3 & 2.6 \\
\hline \multicolumn{7}{|l|}{ Watermaze probe trial } \\
\hline \multirow[t]{2}{*}{ Passes through target area } & 4 & 2.9 & 42 & 0.9 & 0.3 & 3.4 \\
\hline & 12 & 2.5 & 28 & -0.8 & 0.4 & 2.9 \\
\hline \multicolumn{7}{|l|}{ Open field } \\
\hline Total distance & 3 & 3.1 & 90 & -3.3 & -2.0 & 4.7 \\
\hline \multirow[t]{2}{*}{ Entries into center } & 7 & 3.1 & 54 & 4.7 & -26.7 & 6.7 \\
\hline & 15 & 3.6 & 12 & 17.7 & -10.3 & 5.9 \\
\hline \multirow[t]{3}{*}{ Time in periphery } & 6 & 2.4 & 0 & 2.2 & -0.3 & 2.9 \\
\hline & 13 & 2.6 & 28 & -2.2 & -2.7 & 4.6 \\
\hline & 19 & 2.2 & 14 & 0.2 & -4.3 & 5.6 \\
\hline \multicolumn{7}{|l|}{ Conditioned avoidance } \\
\hline Escapes & 7 & 2.6 & 44 & -0.8 & -1.7 & 6.7 \\
\hline Avoidances & 1 & 2.4 & 80 & 0.0 & -1.0 & 3.4 \\
\hline
\end{tabular}

LOD scores of 2.0 and greater are shown; for some phenotypes (for example time spent in the target quadrant during the water maze probe trial), all LOD scores were $<2$, and so no results are shown. The position (Pos.) on the chromosome (Chr) of the LOD score is shown in centimorgans. The effect of the QTL is given under both additive and dominance models. The last column gives the percentage of the total phenotypic variance attributable to the QTL.

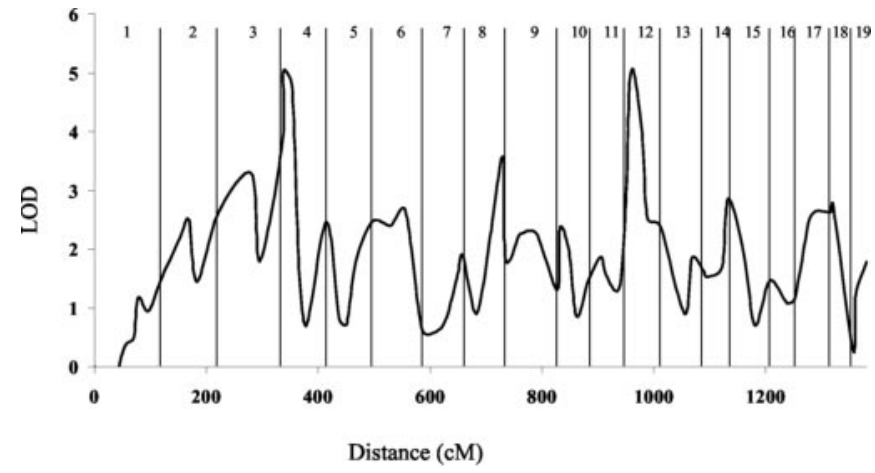

Figure 4. LOD plot for a multivariate analysis of measures taken on the probe trial of the Morris water maze. The vertical axis scale is LOD units ( $2 \mathrm{df}$ ). The horizontal axis is in centimorgans, and each chromosome is shown demarcated by a vertical line with the number of the chromosome written at the top of the graph.

creased over the $9 \mathrm{~d}$ for both latency and distance traveled. Although the maximum observed for the distance traveled is on day 6 , this is not significantly different from the day 9 value. There is also a change in the LOD scores for an effect on chromosome 1 , which shows a peak on day 5 of the experiment (LOD score of 2.3 for the path traveled and 3.8 for latency).

\section{QTL locations on chromosomes 4 and 12}

To obtain the best estimates of the location of the QTL on chromosomes 4 and 12 we used composite interval mapping (Zeng,
Table 2. LOD scores exceeding a 5\% significance threshold in multivariate analyses

\begin{tabular}{lrrrrl}
\hline Phenotype & Chrom. & Pos. & LOD & df & Sig. \\
\hline Watermaze: probe trial & 4 & 32 & 3.5 & 2 & 0.02 \\
& 12 & 25 & 3.2 & & 0.02 \\
Watermaze: $t$ statistics & 4 & 42 & 2.5 & 2 & 0.05 \\
& 8 & 76 & 2.7 & & 0.045 \\
Watermaze: probe trial and & 12 & 23 & 2.9 & & 0.04 \\
$\quad 4$ & 42 & 5.0 & 4 & 0.017 \\
t statistics & 12 & 22 & 5.1 & & 0.015 \\
Open field & 3 & 71 & 3.8 & 3 & 0.027 \\
& 7 & 55 & 5.0 & & 0.01 \\
& 15 & 13 & 4.0 & & 0.015 \\
Conditioned avoidance & 19 & 0 & 4.1 & & 0.017 \\
Open field and conditioned & 1 & 87 & 4.7 & 3 & 0.027 \\
avoidance & 1 & 87 & 5.4 & 6 & 0.048 \\
& 7 & 53 & 6.6 & & 0.009 \\
& 15 & 8 & 5.3 & & 0.043 \\
& 19 & 8 & 6.8 & & 0.011 \\
\hline
\end{tabular}

The table shows the chromosome on which the QTL is found (Chrom.), the position in centimorgans (Pos.), the LOD score with degrees of freedom (df), and the genome-wide significance level (Sig). The combination of traits used in each phenotype is described in Results.

1994). On chromosome 4 we obtained a distinct peak at $46 \mathrm{cM}$ (Fig. 7), but composite interval mapping did not help localize the effect on chromosome 12. Because the effect sizes are small, the location is imprecise: the locus on chromosome 4 accounts for $3.4 \%$ and the QTL on chromosome 12 only $2.9 \%$ of the variance of the joint phenotype. Consequently the $95 \%$ confidence intervals 


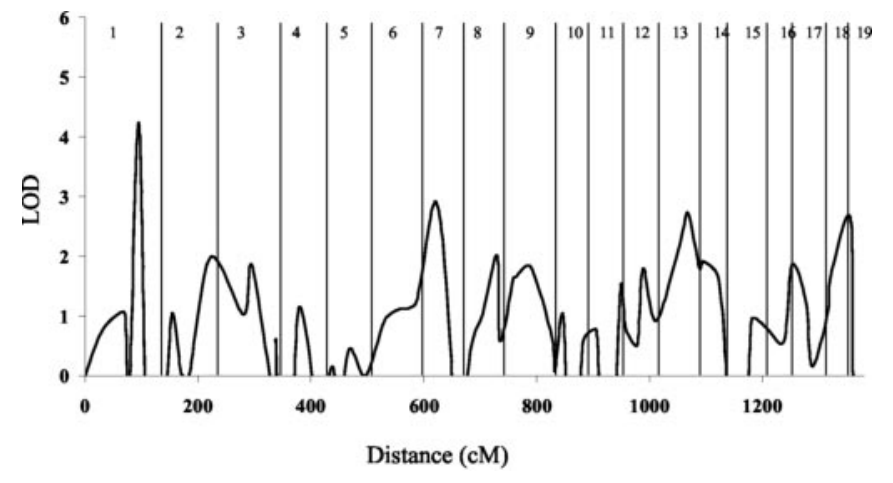

Figure 5. LOD plot for a multivariate analysis of avoidance conditioning. The vertical axis scale is LOD units ( $3 \mathrm{df}$ ). The horizontal axis is in centimorgans, and each chromosome is shown demarcated by a vertical line with the number of the chromosome written at the top of the graph.

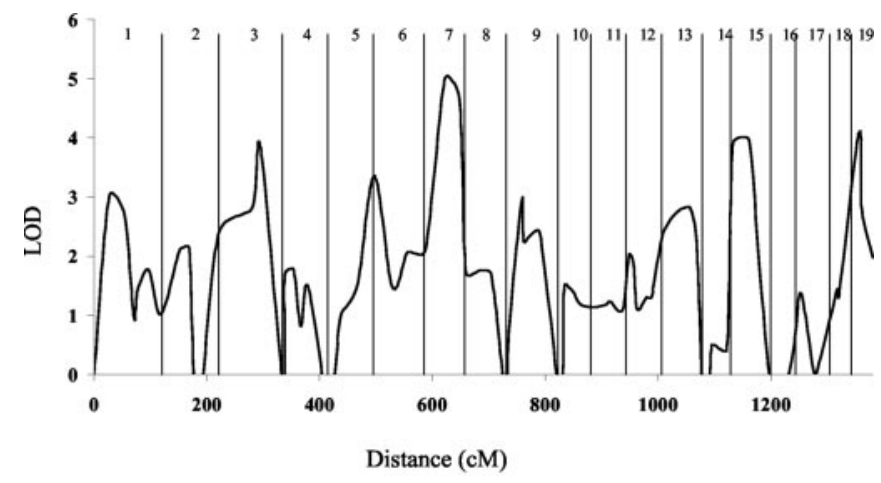

Figure 6. LOD plot for a multivariate analysis of open-field behaviors. The vertical axis scale is LOD units ( $3 \mathrm{df}$ ). The horizontal axis is in centimorgans, and each chromosome is shown demarcated by a vertical line with the number of the chromosome written at the top of the graph.

are wide. Estimated using a bootstrap procedure (Visscher et al., 1996), the confidence interval on chromosome 4 was between 0 and $56 \mathrm{cM}$, and on chromosome 12 it was between 19 and $58 \mathrm{cM}$.

\section{Interaction analysis}

We looked for interactions between QTL on each phenotype using the test advocated by Shimomura et al. (2001). We fitted a linear model to the data and compared the likelihood of a full model including two main effects (for the two loci) and an interaction term with a null model in which there is no genetic effect. For genetic effects discovered to be significant at a 5\% threshold (determined by a permutation test), we then compared the full model with a model that contained only two main effects. We looked to determine whether this test was significant at a nominal $\alpha$ of 0.01 (Shimomura et al., 2001). None of the pairs of loci examined met the requisite criteria. We then tested all pairs in which the full model exceeded a 10\% significance threshold. Even with this relaxed criterion, we obtained no evidence for interaction.

\section{Discussion}

We have performed a genetic mapping experiment of spatial learning in rodents and, using multivariate analyses and permutation tests, provide evidence that two loci, on chromosomes 4 (genome-wide significance $p=0.017$ ) and 12 (genome-wide significance $p=0.015$ ), contribute to genetic variation in performance on the probe trial of the Morris water maze task. Our data indicate that the same loci contribute to variation in how well each mouse learns the spatial task, as assessed by a regression analysis of the distance traveled and latency to reach the platform during the training period. QTL on chromosomes 4,12 , and 8 contribute to variation in the regression-derived $t$ statistics. Analysis of the contribution of the traits to the LOD scores shows that both the number of times an animal passes through the location of the hidden platform on the probe trial and the $t$ statistic for distance traveled during training contribute significantly to the LOD score on chromosome 4, suggesting that this QTL has an effect on both measures.

In addition to measures of spatial learning in the water maze, we looked for a series of possible confounds, including activity and emotional variables. We found a complete separation in genetic effects between tests of activity in a novel environment (QTL on chromosomes 3, 7, 15, and 19), conditioned avoidance (chromosome 1), and spatial learning (chromosomes 4 and 12). Attempts to detect small genetic effects common to these tasks were unsuccessful, but the power of the analyses was insufficient to prove that the QTL on chromosomes 4 and 12 were specific to spatial learning.

Consideration of the way genetic effects operated over the training period in the water maze, however, corroborated the view that the QTL for recall of spatial information were distinct from those that operate during the acquisition of spatial information during the training period. We detected a QTL on chromosome 1 during the early part of the training in the same location as the QTL that influences avoidance training. This locus also lies in the same position as a number of QTL that influence fearful or anxious behavior in the mouse (Caldarone et al., 1997; Gershenfeld and Paul, 1997; Wehner et al., 1997; Turri et al., 2001a). Although our data cannot confirm that it is the same molecular variant, the coincident location indicates that genetic effects on fearful behavior are acting in the training period. In fact, genetic effects on spatial learning are not noticeable until the probe trial. Measures of learning taken during the early part of the training period do not have the same genetic basis as those taken at the end of the trial. Furthermore, using estimates of the contribution of a trait to a LOD score, our data indicate that the locus on chromosome 1 that influences latency to find the hidden platform acts also on a conditioned avoidance task. We suggest therefore that the chromosome 1 QTL reflects the action of fear response that motivates spatial learning, rather than spatial learning per se.

A number of authors have demonstrated that multitrait analysis can improve the detection of quantitative-trait loci with effects that are too small to be found in single-trait analyses (Amos et al., 1990; Schork et al., 1993; Jiang and Zeng, 1995; Korol et al., 1995; Ronin et al., 1995; Mangin et al., 1998). Enhanced sensitivity to detection of an effect arises when a locus has pleiotropic effects that operate in the same direction on each trait included in the analysis, thereby increasing the effect attributable to the locus. Most power obtains when the environmental correlation works in the opposite direction to the genetic effect (Jiang and Zeng, 1995). It follows that there is little or no gain in power when analyzing uncorrelated traits. Here we have demonstrated the value of multivariate techniques to detect small effects, but it should be noted that potential gains from joint consideration of the correlated traits is offset by an increase in the number of parameters, and hence degrees of freedom, which increase the critical value of the test statistic required to achieve a given level of statistical significance (Mangin et al., 1998).

All of the genetic effects that we found were small, detectable only at $5 \%$ significance thresholds using multivariate approaches. 
Table 3. LOD scores for the distance traveled on each day of the water maze test

\begin{tabular}{|c|c|c|c|c|c|c|c|c|c|}
\hline Chr. & Day 1 & Day 2 & Day 3 & Day 4 & Day 5 & Day 6 & Day 7 & Day 8 & Day 9 \\
\hline 1 & 1.4 & 0.3 & 1.2 & 1.4 & 2.3 & 0.9 & 1.5 & 0.5 & 0.3 \\
\hline 2 & 1.2 & 0.9 & 0.8 & 2.2 & 1.0 & 1.6 & 1.1 & 1.4 & 1.9 \\
\hline 3 & 0.8 & 0.6 & 1.6 & 0.4 & 0.7 & 0.4 & 3.6 & 0.5 & 1.0 \\
\hline 4 & 1.6 & 1.5 & 1.7 & 1.0 & 2.5 & 2.8 & 2.1 & 1.9 & 2.7 \\
\hline 5 & 4.2 & 1.4 & 0.6 & 0.8 & 1.3 & 1.7 & 1.2 & 1.0 & 1.4 \\
\hline 6 & 2.4 & 1.1 & 1.6 & 0.4 & 0.3 & 0.6 & 1.4 & 0.5 & 0.6 \\
\hline 7 & 0.2 & 0.8 & 0.1 & 0.7 & 0.3 & 1.0 & 1.4 & 0.4 & 0.1 \\
\hline 8 & 2.0 & 0.8 & 1.1 & 0.8 & 0.8 & 1.8 & 0.9 & 1.1 & 0.6 \\
\hline 9 & 0.5 & 0.6 & 2.1 & 0.5 & 1.9 & 2.2 & 1.7 & 1.2 & 0.8 \\
\hline 10 & 1.6 & 1.3 & 1.4 & 1.5 & 2.8 & 0.9 & 2.9 & 1.2 & 0.5 \\
\hline 11 & 1.2 & 1.2 & 1.8 & 0.3 & 0.3 & 0.9 & 0.1 & 0.5 & 0.2 \\
\hline 12 & 0.6 & 1.3 & 0.6 & 1.1 & 0.4 & 1.3 & 1.2 & 1.4 & 2.4 \\
\hline 13 & 1.6 & 0.7 & 0.3 & 0.3 & 0.8 & 0.4 & 1.0 & 1.1 & 0.8 \\
\hline 14 & 2.3 & 0.6 & 0.3 & 0.4 & 0.8 & 0.6 & 0.8 & 1.1 & 1.6 \\
\hline 15 & 1.3 & 1.8 & 1.0 & 1.2 & 0.5 & 1.9 & 1.6 & 1.2 & 0.3 \\
\hline 16 & 0.5 & 0.4 & 0.5 & 0.5 & 2.0 & 0.9 & 0.2 & 0.6 & 0.2 \\
\hline 17 & 1.8 & 0.7 & 0.4 & 0.6 & 0.5 & 0.6 & 0.1 & 1.6 & 0.8 \\
\hline 18 & 0.3 & 1.1 & 1.4 & 0.7 & 0.1 & 0.4 & 0.7 & 0.3 & 1.4 \\
\hline 19 & 1.5 & 1.0 & 1.1 & 0.6 & 0.9 & 0.6 & 0.2 & 0.7 & 0.8 \\
\hline
\end{tabular}

The table shows the maximum LOD score obtained on each chromosome (Chr.) for each day.

Table 4. LOD scores for latency to reach the platform on each day of the water maze test

\begin{tabular}{|c|c|c|c|c|c|c|c|c|c|}
\hline Chr. & Day 1 & Day 2 & Day 3 & Day 4 & Day 5 & Day 6 & Day 7 & Day 8 & Day 9 \\
\hline 1 & 1.9 & 3.5 & 1.7 & 2.6 & 3.8 & 2.2 & 2.3 & 1.1 & 1.2 \\
\hline 2 & 1.0 & 0.8 & 0.6 & 1.2 & 1.8 & 1.0 & 1.3 & 1.1 & 1.3 \\
\hline 3 & 0.4 & 0.7 & 1.4 & 0.4 & 0.6 & 0.7 & 0.3 & 0.8 & 0.8 \\
\hline 4 & 1.0 & 1.6 & 0.5 & 1.1 & 1.4 & 1.3 & 1.6 & 1.3 & 2.3 \\
\hline 5 & 0.4 & 0.9 & 0.8 & 0.8 & 0.4 & 0.3 & 0.7 & 0.3 & 0.5 \\
\hline 6 & 0.3 & 1.2 & 1.0 & 0.4 & 1.2 & 0.8 & 1.3 & 0.6 & 1.1 \\
\hline 7 & 1.5 & 1.1 & 0.4 & 0.5 & 1.1 & 1.6 & 1.4 & 1.0 & 0.5 \\
\hline 8 & 0.5 & 0.5 & 0.6 & 0.5 & 1.2 & 1.0 & 0.6 & 0.9 & 1.1 \\
\hline 9 & 1.3 & 1.1 & 1.3 & 0.3 & 0.7 & 0.5 & 0.6 & 0.9 & 0.3 \\
\hline 10 & 1.9 & 2.7 & 1.2 & 1.7 & 4.0 & 2.3 & 2.4 & 1.9 & 1.9 \\
\hline 11 & 2.1 & 1.9 & 1.3 & 0.6 & 0.2 & 1.6 & 0.5 & 0.9 & 0.2 \\
\hline 12 & 0.4 & 0.5 & 0.8 & 1.6 & 0.8 & 1.6 & 2.2 & 1.4 & 2.3 \\
\hline 13 & 0.7 & 1.1 & 1.4 & 0.5 & 1.2 & 1.6 & 2.1 & 2.5 & 2.5 \\
\hline 14 & 0.7 & 0.3 & 0.4 & 0.2 & 0.7 & 0.3 & 0.4 & 0.9 & 0.6 \\
\hline 15 & 1.9 & 1.5 & 1.3 & 0.9 & 0.5 & 1.0 & 0.9 & 1.3 & 0.4 \\
\hline 16 & 2.0 & 0.2 & 0.7 & 0.2 & 0.9 & 0.4 & 0.4 & 0.9 & 0.5 \\
\hline 17 & 0.8 & 0.9 & 0.5 & 1.1 & 0.5 & 0.9 & 0.3 & 1.0 & 0.9 \\
\hline 18 & 0.3 & 0.7 & 1.9 & 1.9 & 1.1 & 1.3 & 0.6 & 0.6 & 0.2 \\
\hline 19 & 0.5 & 1.2 & 1.1 & 0.5 & 1.2 & 1.1 & 0.3 & 0.5 & 0.8 \\
\hline
\end{tabular}

The table shows the maximum LOD score obtained on each chromosome (Chr.).

We found no evidence of epistatic effects acting on any of the phenotypes investigated, so we believe we have not missed any additional sources of genetic variation. The small size of the genetic effects means that the $95 \%$ confidence intervals are broad: the QTL on chromosomes 12 and 4 are contained in intervals of $\sim 50 \mathrm{cM}$. Despite this, we were surprised to discover that the broad confidence intervals do not contain genes known, from transgenic analysis, to influence spatial learning. Using the mouse genome sequence, we located genes listed by (Sanes and Lichtman, 1999) and found that none were on either chromosome 4 or 12 (Table 5). This result suggests that the QTL contain genes not so far known to be involved in spatial learning.

Our results complement those of the first paper to report genetic mapping of spatial learning (Milhaud et al., 2002). The latter study used recombinant inbred (RI) lines in which a QTL on chromosome 1 was found to influence latency in the training phase of the water maze and a QTL on chromosome 5 was found to influence the probe trial behavior (Milhaud et al., 2002). As argued above, and in agreement with Milhaud and colleagues (2002), we believe that the locus on chromosome 1 modulates fearful behavior and not spatial learning. Although our data replicate the finding of the chromosome 1 QTL influencing latency, we did not find a QTL on chromosome 5. The RI lines were derived from DBA/2 and C57BL/6, and so we cannot attribute the disagreement in findings to the segregation of different alleles in the two studies. Because the effects that we have detected are small, it is possible that our study failed to detect a QTL on chromosome 5, or that the discrepancy between our work and that of the recombinant inbred study is attributable to differences in phenotyping, or that the RI result is a false positive. John 


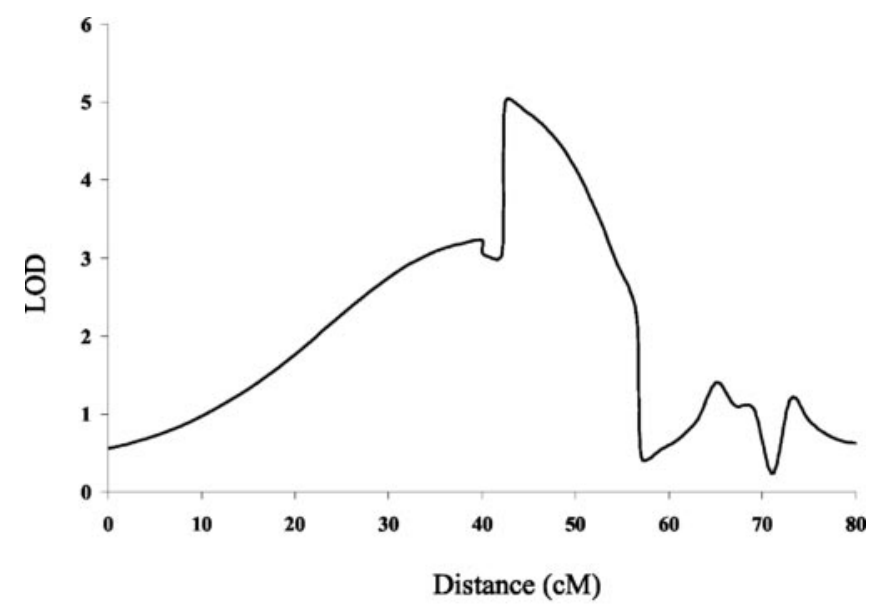

Figure 7. Composite interval mapping a QTL that influences spatial learning on chromosome 4. The vertical scale is LOD units (4 df). The horizontal scale is in centimorgans.

\begin{tabular}{|c|c|c|c|}
\hline Gene & Chromosome & Gene & Chromosome \\
\hline GLUR2 & 3 & PSD95 & 11 \\
\hline MGLUR1 & 11 & $R A R \beta$ & 14 \\
\hline MGLUR4 & 9 & CREB & 15 \\
\hline MGLUR5 & 16 & KROX 24 & 18 \\
\hline MAS proto-oncogene & 17 & KROX 20 & 10 \\
\hline NMDA NR1 & 2 & NCAM 1 & 9 \\
\hline NMDA NR2A & 16 & NCAM 2 & 16 \\
\hline NMDA NR2D & 7 & $\mathrm{~N}$-cadherin & 18 \\
\hline$\beta$-Adrenergic receptor & 18 & E-cadherin & 8 \\
\hline Adenosine $A 2 B$ receptor & 11 & $T H Y-1$ & 9 \\
\hline Adenosine A2A receptor & 10 & $\begin{array}{l}\text { L1 CAM } \\
\text { Inositol 1,4,5 trisphosphate } 3\end{array}$ & $x$ \\
\hline Dopamine D1A receptor & 13 & kinase & 7 \\
\hline Nocicetin receptor & 2 & Tyrosine-protein kinase FYN & 10 \\
\hline BDNF & 2 & Activator & 8 \\
\hline Calretinin & 8 & Ubiquitin-protein ligase E3A & 7 \\
\hline $\begin{array}{l}\text { Olfactory cyclic nucleotide- } \\
\text { gated channel }\end{array}$ & $X$ & Phospholipase A2 & 10 \\
\hline$R A B 3 A$ & 8 & $\begin{array}{l}\text { Adenylyl cyclase type I } \\
\text { Glial fibrillary acidic protein, } \\
\text { astrocyte }\end{array}$ & 11 \\
\hline
\end{tabular}

Belknap and colleagues (1996) have shown that only approximately half of the QTL identified using RI strains represent true associations.

Our mapping results for conditioned avoidance and openfield activity replicate the findings of other groups who have mapped QTL influencing fear conditioning to chromosomes 1,2, 3, 10, and 16 (Wehner et al., 1997). We have been able to detect the QTL on chromosome 1, but other loci were below genomewide significance. There have been no studies of open-field behavior in a C57BL/6 by DBA/2 cross, but, intriguingly, QTL on chromosomes 3, 7, 15, and 19 have all been detected in other crosses (Gershenfeld et al., 1997; Turri et al., 2001a). It is particularly noteworthy that we have detected a locus in the middle of chromosome 7 , which replicates that found in a cross between inbred mice with alleles derived from C57BL/6 and BALBc/J (Turri et al., 2001b). The effect on chromosome 7 is believed to be attributable to a tyrosinase mutation (the clocus that gives rise to albinism), because a number of investigators have attested to the timidity of albino mice (DeFries et al., 1966; Henry and Schlesinger, 1967; DeFries, 1969). The animals that we used do not carry the tyrosinase mutation (data not shown), so we assume that the effect is attributable to a locus somewhere else on chromosome 7 .

In summary, we have detected two QTL, on chromosomes 4 and 12, that influenced behavior in the probe trial of the Morris water maze. By including tests of avoidance conditioning and behavior in a novel environment, we have accumulated evidence that indicates that the QTL effects are specific to spatial learning, although our study does not have sufficient power to exclude pleiotropic action.

\section{References}

Amos CI, Elston RC, Bonney GE, Keats BJB, Berenson GS (1990) A multivariate method For detecting genetic-linkage, with application to a pedigree with an adverse lipoprotein phenotype. Am J Hum Genet 47:247-254.

Basten CJ, Weir BS, Zeng Z-B (1994) QTL Cartographer: a suite of programs for mapping quantitative trait loci. In: Proceedings of the 5 th World Congress on genetics applied to livestock production: computing strategies and software (Smith C, Gavora JS, Chesnais BBJ, Fairfull W, Gibson JP, Kennedy BW, Burnside EB, eds), pp. 65-66. Ontario: Guelph.

Belknap JK, Mitchell SR, O’Toole LA, Helms ML, Crabbe JC (1996) Type I and type II error rates for quantitative trait loci (QTL) mapping studies using recombinant inbred mouse strains. Behav Genet 26:149-160.

Caldarone B, Saavedra C, Tartaglia K, Wehner JM, Dudek BC, Flaherty L (1997) Quantitative trait loci analysis affecting contextual conditioning in mice. Nat Genet 17:335-337.

Chen C, Tonegawa S (1997) Molecular genetic analysis of synaptic plasticity, activity-dependent neural development, learning, and memory in the mammalian brain. Annu Rev Neurosci 20:157-184.

Contet C, Rawlins JN, Bannerman DM (2001) Faster is not surer-a comparison of C57BL/6J and 129S2/Sv mouse strains in the water maze. Behav Brain Res 125:261-267.

Crawley JN, Belknap JK, Collins A, Crabbe JC, Frankel W, Henderson N, Hitzemann RJ, Maxson SC, Miner LL, Silva AJ, Wehner JM, WynshawBoris A, Paylor R (1997) Behavioral phenotypes of inbred mouse strains: implications and recommendations for molecular studies. Psychopharmacology (Berl) 132:107-124.

Dawson GR, Seabrook GR, Zheng H, Smith DW, Graham S, O’Dowd G, Bowery BJ, Boyce S, Trumbauer ME, Chen HY, Van Der Ploeg LHT, Sirinathsinghji DJS (1999) Age-related cognitive deficits, impaired long-term potentiation and reduction in synaptic marker density in mice lacking the $\beta$-amyloid precursor protein. Neuroscience 90:1-13.

DeFries JC (1969) Pleiotropic effects of albinism on open field behaviour in mice. Nature 221:65-66.

DeFries JC, Hegmann JP, Weir MW (1966) Open-field behavior in mice: evidence for a major gene effect mediated by the visual system. Science 154:1577-1579.

D'Hooge R, De Deyn PP (2001) Applications of the Morris water maze in the study of learning and memory. Brain Res Brain Res Rev 36:60-90.

Fernandez-Teruel A, Escorihuela RM, Gray JA, Aguilar R, Gil L, GimenezLlort L, Tobena A, Bhomra A, Nicod A, Mott R, Driscoll P, Dawson GR, Flint J (2002) A quantitative trait locus influencing anxiety in the laboratory rat. Genome Res 12: 618-626.

Flint J, Mott R (2001) Finding the molecular basis of quantitative traits: successes and pitfalls. Nat Rev Genet 2:438-445.

Gershenfeld HK, Paul SM (1997) Mapping quantitative trait loci for fearlike behaviors in mice. Genomics 46:1-8.

Gershenfeld HK, Neumann PE, Mathis C, Crawley JN, Li X, Paul SM (1997) Mapping quantitative trait loci for open-field behavior in mice. Behav Genet 27:201-210.

Gray JA, McNaughton N (1983) Comparison between the behavioural effects of septal and hippocampal lesions: a review. Neurosci Biobehav Rev 7:119-188.

Henry KR, Schlesinger K (1967) Effects of the albino and dilute loci on mouse behavior. J Comp Physiol Psychol 63:320-323.

Holscher C (1999) Stress impairs performance in spatial water maze learning tasks. Behav Brain Res 100:225-235. 
Jiang C, Zeng Z-B (1995) Multiple trait analysis of genetic mapping for quantitative trait loci. Genetics 140:1111-1127.

Korol AB, Ronin YI, Kirzhner VM (1995) Interval mapping of quantitative trait loci employing correlated trait complexes. Genetics 140:1137-1147.

Korol AB, Ronin YI, Itskovich AM, Peng J, Nevo E (2001) Enhanced efficiency of quantitative trait loci mapping analysis based on multivariate complexes of quantitative traits. Genetics 157:1789-1803.

Lincoln S, Daly M, Lander E (1992) Mapping genes controlling quantitative traits with MAPMAKER/QTL 1.1. Whitehead Institute Technical Report, Cambridge, MA.

Mangin B, Thoquet P, Grimsley N (1998) Pleiotropic QTL analysis. Biometrics 54:88-99.

Milhaud JM, Halley H, Lassalle JM (2002) Two QTLs located on chromosomes 1 and 5 modulate different aspects of the performance of mice of the BxDTy RI strain series in the Morris navigation task. Behav Genet 32:69-78.

Ronin YI, Kirzhner VM, Korol AB (1995) Linkage between loci of quantitative traits and marker loci-multi-trait analysis with a single marker. Theo Appl Genet 90:776-786.

Sanes JR, Lichtman JW (1999) Can molecules explain long-term potentiation? Nat Neurosci 2:597-604.
Schork NJ, Boehnke M, Terwilliger JD, Ott J (1993) Two-trait-locus linkage analysis: a powerful strategy for mapping complex genetic traits. Am J Hum Genet 53:1127-1136.

Shimomura K, Low-Zeddies SS, King DP, Steeves TD, Whiteley A, Kushla J, Zemenides PD, Lin A, Vitaterna MH, Churchill GA, Takahashi JS (2001) Genome-wide epistatic interaction analysis reveals complex genetic determinants of circadian behavior in mice. Genome Res 11:959-980.

Turri MG, Datta SR, DeFries J, Henderson ND, Flint J (2001a) QTL analysis identifies multiple behavioral dimensions in ethological tests of anxiety in laboratory mice. Curr Biol 11:725-734.

Turri MG, Henderson ND, DeFries JC, Flint J (2001b) Quantitative trai locus mapping in laboratory mice derived from a replicated selection experiment for open-field activity. Genetics 158:1217-1226.

Visscher PM, Thompson R, Haley CS (1996) Confidence-intervals in QTL mapping by bootstrapping. Genetics 143:1013-1020.

Wehner JM, Radcliffe RA, Rosmann ST, Christensen SC, Rasmussen DL, Fulker DW, Wiles M (1997) Quantitative trait locus analysis of contextual fear conditioning in mice. Nat Genet 17:331-334.

Zeng Z-B (1994) Precision mapping of quantitative trait loci. Genetics 136: 1457-1468. 